\title{
THE STUDY OF PASSIVE VIBRATION DAMPERS IN PIPELINES USING PIV-METHODOLOGY FOR SINGLE PHASE FLOW
}

\author{
ISMAIL HOSSAIN ${ }^{1,2}$, VLADIMIR I. VELKIN ${ }^{1} \&$ SERGEI E. SHCHEKLEIN ${ }^{1}$ \\ ${ }^{1}$ Department of Nuclear Power Plants and Renewable Energy, Ural Federal University, Yekateringburg, Russia \\ ${ }^{2}$ Department of Physics, University of Dhaka, Bangladesh
}

\begin{abstract}
An experimental investigation of the study for pulsed particle visualization of micro tracers using the PIV technique was conducted. The purpose of the study was to obtain the velocity distribution in straight and turns of the pipeline and influence of different geometries of the inserts (swirlers) on the cause of the pressure gradient on the outer and inner walls of the parts. The visualization of fluid flow consisting tracer particles was determined. In order to study the flow pattern a design of passive devices was performed and put into experimentation to investigate the flow field. The PIV-technique and equipment allow the analytical study of the microstructure for hydrodynamic fluid flow and the measurement of piping vibration after passing through the passive damper. It was found that the use of the improved devices leads to the reduction of piping vibration at $25-50 \%$. It was possible to figure out the velocity distribution pattern as well as the visualization of the swirling of the fluid flow based on PIV technique.
\end{abstract}

Keywords: PIV methodology, pressure gradient, velocity distribution, single phase flow, piping vibration.

\section{INTRODUCTION}

In this study, we investigate a liquid flow with micro tracers in order to understand the study of motion and turbulence of the flow through a vertical pipe. The flow with turbulence along with the orientation of tracer particles and their inter connection with the liquid generates a complex behaviour consisting the function Reynolds number $\left(\mathrm{R}_{\mathrm{e}}\right)$, particle Reynolds number $\left(R_{e p}\right)$ and Stokes number $\left(S_{t}\right)$, particle/fluid density ratio $\left(\rho_{p} / \rho_{f}\right)$, flow orientation, and solid phase volumetric concentration $\left(\phi_{\mathrm{v}}\right)$. With the addition of passive inserts of different geometrical shapes this system shows different gesture of the flow pattern. Particles bear substantial consequences on the transport properties of the mixture for turbulent fluid flow [1]. Among all, slug flow is on the top for its large number of practicable uses. Different power plants possess this kind of flow in vapour-liquid absorbers, vapour generators, reboilers, buoyancy-driven fermenters and vaporizers. The same kind of flow is also recognized in emergency core cooling of nuclear power stations, Oil extraction process, hydrocarbon transfer system in a pipe and in thermal power stations [2]. Kussin and Sommerfield [3] sorted out particle turbulence intensities for particulate gas flow in horizontal pipeline and pointed that the wall roughness had a defective impact on the turbulence intensity of the particle. In case of thermal and nuclear power stations the operation system of comparative equipment is somehow associated with the reduction of vibration level in the pipeline. Excessive vibration in the pipeline due to two phase flow increases the cost of the sustainment, demeans the liableness of the energy system, and also causes serious fatigue failure of metal due to the depressurization of the coolant flow [4]. By changing in the different geometrical shapes of passive inserts it is possible to bring down the vibration level and other hydrodynamic problems in a certain amount [5]. Two phase flow research can bring forth new solutions and facilities in case of fluid dynamics and equipment design basis. It is somehow not possible to eliminate all the problems at a time but it will 
generate new ways to solution of multi problems due to flow pulsations [6]. Kameyama et al. [7] implemented PIV system to obtain results for turbulent variations of water and glass beads in bth direction downword and upword pipe flow for renoylds number 19500. From the study of Suzuki et al. [8] it was noted that the particle and the carrier phase turbulence for $0.4 \mathrm{~mm}$ ceramic beads and water in downward channel flow at $\mathrm{Re}=7500$ using 3D-PTV. To get further insight into the underlying flow physics, the velocity field is decomposed into the mean flow, the wave-induced flow, and the turbulent fluctuations [9].

\section{THEORETICAL APPROACH TO VORTEX INSERTS}

Determination of the twist intensity, the kinetic energy ratio of the whirling part of the flow and the energy of the translational (axial) flow can be represented as follows:

$$
\varepsilon=(\operatorname{tg} \varphi)^{2} \mathrm{nf} / \mathrm{F}_{0} ; \operatorname{tg} \varphi=\mathrm{U}_{\varphi} / \mathrm{U}_{0},
$$

where $\mathrm{n}$ - is the number of swirl grooves; f and F0 - are the values of the total cross-sectional area of the grooves and the cylindrical channel; $\mathrm{U} \varphi$ and $\mathrm{U}_{0}$ - are the tangential and axial flow velocities. Darcy-Weisbach equation leads a solution to the hydrodynamic features of the vortex inserts for single phase flow from pressure drop measurements:

$$
\xi=2(\mathrm{~d} / \mathrm{L})\left(\Delta \mathrm{p} / \rho \mathrm{U}_{\mathrm{o}}^{2}\right),
$$

where $\xi$ - is the coefficient of hydraulic resistance (CHR) of the insert; $d$ and L - are the hydraulic diameter and length of the insert.

\section{EXPERIMENTAL SET-UP AND MEASURING TECHNIQUE}

To carry on the experiment, we set up an arrangement of closed pipeline system conjoined a circulation pump. This closed circulating flow scheme consists of four sections: a horizontal section with transition to the ascending vertical line, the same as a vertical ascending with a transition to horizontal, a horizontal with transition to the descending vertical and a vertical lowering with transition to horizontal component. The circulation speed is adjustable from 2 to $6 \mathrm{~m} / \mathrm{s}$. In an experimental section of the PIV experiments were conducted within the transparent Plexiglas pipe of internal diameter $\mathrm{D}=45 \mathrm{~mm}$. The pipe consisted of smooth grooved passive inserts of length $\mathrm{L}=30 \mathrm{~cm}$, diameter $\mathrm{d}=45 \mathrm{~mm}$ and number of grooves $\mathrm{n}=18$. The pipe also comprised of adjacent sections connected by annular joints of stiff tightness. All joints were firmly attached by collars to vertical and horizontal beams that held up the entire structure. In order to pursue the research, we managed to build up an experimental structure to allow the fluid flow with some micro tracers and we were able to set a PIV experimental arrangement (Fig. 1).

After a number of tests, the study provides a clear anticipation of design improvement and probable suitability in the reduction of piping vibration [10]. Most importantly, this device has low hydraulic resistance and has the availability to adjust as series connection with pipeline. Within a transparent Plexiglas sections the investigation of swirling effect of the fluid was accomplished [11]. Fig. 2 shows vortex inserts with correctional grooves depiction. 


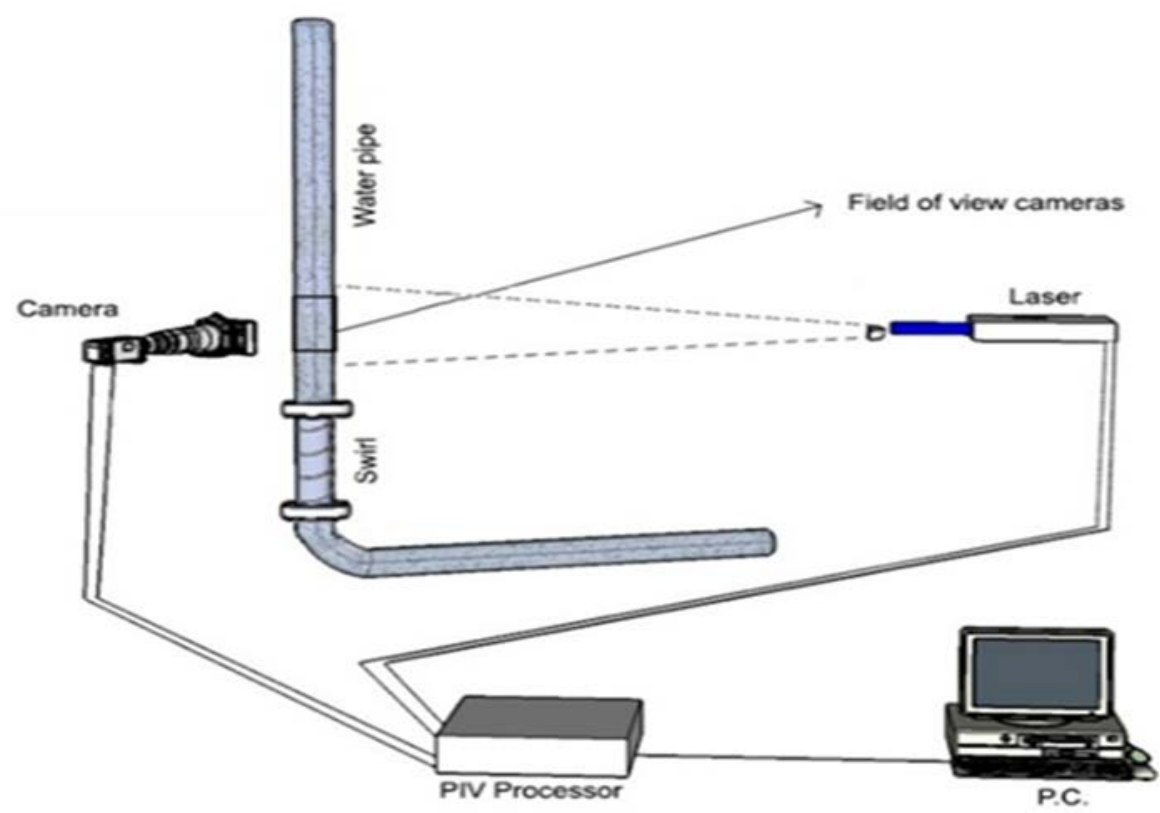

Figure 1: Representation of PIV components around the experimental section.

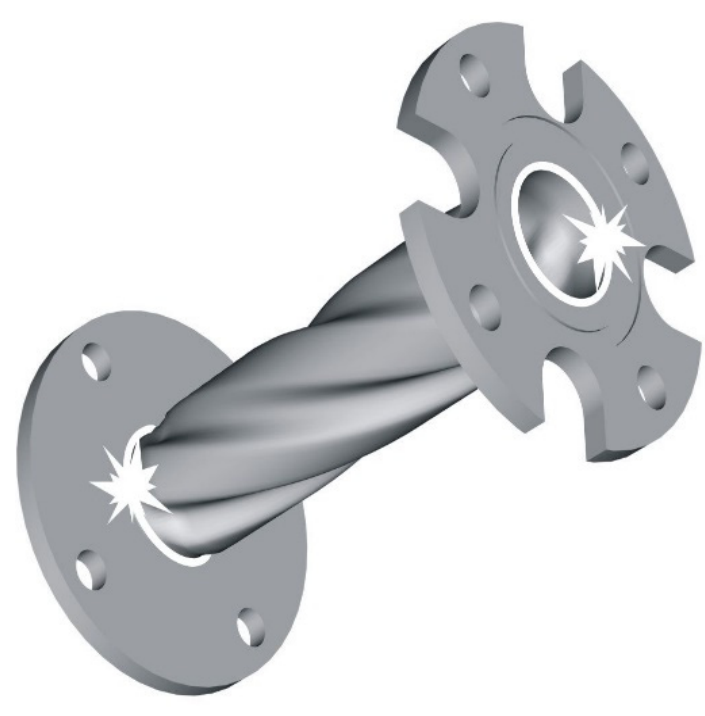

(a)

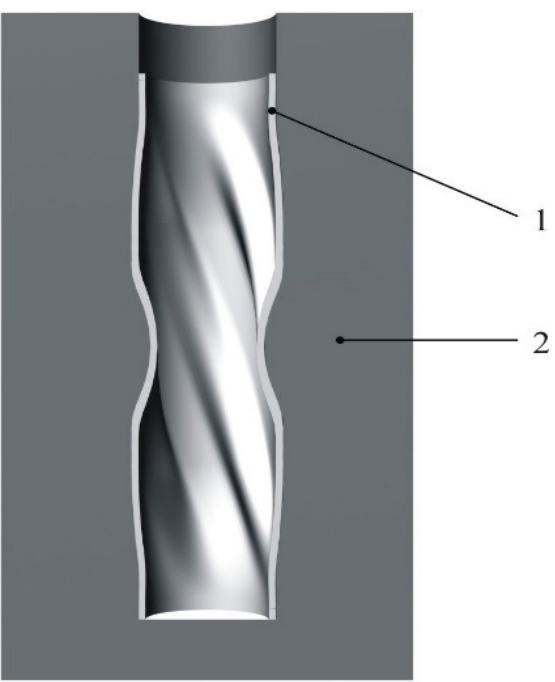

(b)

Figure 2: (a) Model scheme of designed passive insert; (b) Cross-section of vortex insert and grooves depiction-1 the workpiece, 2 the mold. 


\section{UTILIZATION OF PASSIVE INSERTS USING THE PIV METHODOLOGY}

The experimental stand was equipped with a laser installation and a receiving camera. For better experimentation, we introduced a very useful visualization technique with combination of high resolution image processing unit called PIV (Particle Image Velocimetry) methodology. In order to record hydrodynamic fluctuations in the liquid flow, micro tracers (plastic powder) with a particle diameter of $90 \mu \mathrm{m}$ were introduced in the circulating piping system. This process appropriated to evaluate the particle velocity field up to $2-5 \mu \mathrm{m} /$ vector associated with liquid flow within the submillimetre range. The visualization of velocity measurement by the computerized image processing system is shown in Fig. 3.

In the investigated section of the stand with inserts-swirlers, the change in flow structure and vibration of the pipeline (vibration displacement, vibration velocity, vibration acceleration) can be measured. Fig. 3(a) shows the visual vector representation influenced by the impact of roughly grooved external passive insert of same size and same number of groves as used for Fig. 3(b) within the same scale. This vector orientation indicates abrupt and unsteady motion of tracer particles. On the contrary, the Fig. 3(b) clarifies comparatively uniform swirling flow as in this case smooth passive device has been used. This is how PIV methodology leads to an improvement for the passive insert design. The stand allows to investigate the influence of swirlers on a single-phase flow (water or air), as well as on two-phase flow. The task of reducing the vibration of pipelines is of paramount importance for improving the reliability of equipment and the safety of energy facilities. Some fluid flow parameters comprising velocity derivative, particular distributions of instantaneous flow velocity and complete measurement of statistical aspects can be experimented using "Polis" system [12]. Along with the experimental arrangement we put into action a High Powered Martin Magnum 1800 tracer device. The installation ensures the pertinence of acquired outcome of fluid flow study within the range $10^{4}-10^{6}$ reynolds number [13], [14]. The PIV system "Polis" improved at the Thermos Physics SB RAS ascertains the measurement of velocity fields in the model experimental section [15], [16]. From the synchronized image processing unit it was able to figure out velocity vector visualization for single phase flow within the experimental section.
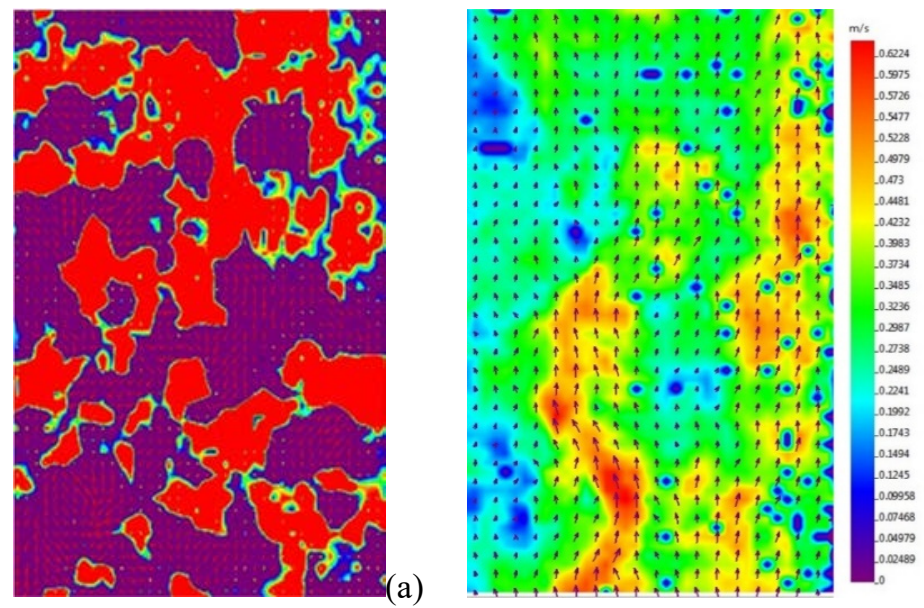

(b)

Figure 3: (a) Velocity measurement within pipeline for rough grooved insert; (b) Visual image for fluid flow swirling of single phase flow through smooth grooved passive insert. 


\section{CONCLUSION}

The decrease in vibrational shift of pipelines was secured by the design and installation of external passive device. A very signified experimental orientation for PIV technology was assembled combined with camera of high resolving power to track the particle used for the test. The system of optical measurement of hydrodynamic features of the coolant flow (PIV-method) assured to obtain the velocity distributions in rectilinear and different turning parts of the pipeline. The test shows a substantial difference of visualization for smooth and rough grooved passive devices. The designed vortex insertion devices reduce the vibration displacement within a significant number of level.

\section{REFERENCES}

[1] Sivakumar, S., Chidambaram, M. \& Shankar, H.S., On the effect of particle size on heat transfer in vertical upflow of gas-solids suspension. Can. J. Chem. Eng., 66, pp. 1000-1004, 1988.

[2] Bugg, J., Mack, K. \& Rezkallah, K., A numerical model of Taylor bubbles rising through stagnant liquids in vertical tubes. Int. J. Multiph. Flow, 24, pp. 271-281, 1998.

[3] Kussin, J. \& Sommerfeld, M., Experimental studies on particle behaviour and turbulence modification in horizontal channel flow with different wall roughness. Exp. Fluids 33, pp. 143-159, 2002.

[4] Baranenko, V.I., Oleinik, S.G., Belyakov, O.A., Istomin, R.S. \& Kumov, A.V., Erosion-corrosion wear of equipment and pipelines at NPPs With PWR and VVER and its impact on Reliability and Safety of Nuclear Power Plants, Fourth International Scientific and Technical Conference. Ensuring the Safety of NPPs with VVR, Podolsk, FSUE OKB GIDROPRESS, 2005.

[5] Fedorovich, E.D., Fokin, B.S. \& Axelrod, A.F., Vibration of equipment elements of nuclear power plants. M: Energoatomizdat, 168, 1989.

[6] Belenky, M.Ya. Gotovsky, M.A. \& Fokin, B.S., Elimination of vibrations of pipelines transporting effervescent and two-phase flows. Teploenergetika, 3, 41-46, 1996

[7] Kameyama, K., Kanai, H., Kawashima, H. \& Ishima, T., Evaluation of particle motion in solid-liquid two-phase pipe flow with downward/upward flow directions. In: 17th International Symposium on Applications of Laser Techniques to Fluid Mechanics, pp. 1-15, 2014.

[8] Suzuki, Y., Ikenoya, M. \& Kasagi, N., Simultaneous measurement of fluid and dispersed phases in a particle-laden turbulent channel flow with the aid of 3-D PTV. Exp. Fluids, 29, pp. 185-193. 2000.

[9] Kemp, P. \& Simons, R.R., The interaction between waves and a turbulent Current: waves propagating with the current. J. Fluid Mech., 116, pp. 227-250, 1982.

[10] Kasina, V.I. \& Leontiev, A.I., Hydraulic resistance of swirling water flows and steamwater mixture in pipes. Heat-power engineering, 3, pp. 40-47, 2005

[11] Velkin, V.I., Komoza, D.S., Krutikov, A.Yu. \& Khnykina, V.V., Microprocessor control unit for complex diagnostic stand for research of vibration of NPP pipelines. News of Higher Educational Establishments “Nuclear Energy”, 1, pp. 4-7, 2009.

[12] Akhmetbekov, E.K., Bilsky, A.V., Markovich, D.M., Maslov, A.A., Polivanov, P.A., Tsyryulnikov, I.S. \& Yaroslavtsev, M.I., The use of the laser measuring complex "POLIS" for measurements of velocity fields in a supersonic flow in wind tunnels .Thermophysics and Aeromechanics. T. 16(3), pp. 343-352, 2009.

[13] Maslov, A.A. \& Mironov, S.G., Experimental investigation of the hypersonic lowdensity flow around a semi-closed cylindrical cavity, Izv. RAS. FGM, 6, pp. 155-160, 1996. 
570 Energy and Sustainability VII

[14] Scarano, F., Overwiew of PIV in supersonic flows. Particle Image Velocimetry, ed. Schroeder, A. \& Willert. C.E., Springer-Verlag, Berlin, Heidelberg, pp. 445-463, 2008.

[15] Tokarev, M.P., Markovich, D.M. \& Bilsky, A.V., Adaptive algorithms for image processing of particles for the calculation of instantaneous velocity fields. Computational technologies. T, 12(3), 109-131, 2007.

[16] Alekseenko, S.V., Bilsky, A.V. \& Markovich, D.M., Application of the digital tracer visualization method for the analysis of turbulent flows with a periodic component. Devices and experimental technique. S. 47(5), pp. 703-710, 2004. 